\title{
Sonic Boom Mitigation Through Aircraft Design and Adjoint Methodology
}

\author{
Sriram K. Rallabhandi* \\ National Institute of Aerospace, Hampton, VA 23666 \\ Eric J. Nielsen ${ }^{\dagger}$ \\ NASA Langley Research Center, Hampton, VA, 23681 \\ Boris Diskin ${ }^{\ddagger}$ \\ National Institute of Aerospace, Hampton, VA 23666
}

\begin{abstract}
This paper presents a novel approach to design of the supersonic aircraft outer mold line (OML) by optimizing the A-weighted loudness of sonic boom signature predicted on the ground. The optimization process uses the sensitivity information obtained by coupling the discrete adjoint formulations for the augmented Burgers Equation and Computational Fluid Dynamics (CFD) equations. This coupled formulation links the loudness of the ground boom signature to the aircraft geometry thus allowing efficient shape optimization for the purpose of minimizing the impact of loudness. The accuracy of the adjoint-based sensitivities is verified against sensitivities obtained using an independent complex-variable approach. The adjoint based optimization methodology is applied to a configuration previously optimized using alternative state of the art optimization methods and produces additional loudness reduction. The results of the optimizations are reported and discussed.
\end{abstract}

\section{Nomenclature}

$1+\frac{\gamma-1}{2}$

Non-dimensional thermo-viscous parameter

1.4

$\Lambda_{b} \quad$ Adjoint variables corresponding to boom equations

$\Lambda_{f} \quad$ Adjoint variables corresponding to flow equations

$\Lambda_{g} \quad$ Adjoint variables corresponding to grid equations

$\lambda_{n}, \beta_{n}, \gamma_{0, n}, \gamma_{1, n}$ Adjoint vectors

$\rho_{0} \quad$ Ambient density

$\sigma \quad$ Non-dimensional distance

$\tau \quad$ Non-dimensional time

$\tau^{\prime} \quad$ Retarded time

G CFD grid equations

Q CFD solution vector

$\mathbf{R} \quad$ CFD discrete flow equations

T Transformation mapping CFD solution to a desired off-body pressure distribution

X Mesh solution vector

$\theta_{/ n u} \quad$ Non-dimensional relaxation time patameter

$A^{n}, B^{n} \quad$ Matrices during the first relaxation process

$A_{2}^{n}, B_{2}^{n} \quad$ Matrices during the second relaxation process

$A_{3}^{n}, B_{3}^{n} \quad$ Matrices during the absorption process

$c_{0} \quad$ Ambient speed of sound, $\mathrm{m} / \mathrm{s}$

$C_{\nu} \quad$ Non-dimensional dispersion

\footnotetext{
*Senior Research Engineer, National Institute of Aerospace, Senior Member AIAA

${ }^{\dagger}$ Research Engineer, Computational AeroSciences Branch, Senior Member AIAA

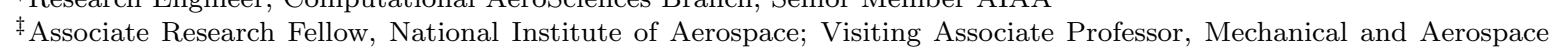
Engineering Dept., University of Virginia, Associate Fellow AIAA
} 


$\begin{array}{ll}D & \text { Vector of design variables } \\ G & \text { Ray Tube Area } \\ I_{N} & \text { Objective/cost function for adjoint calculation } \\ k_{n} & \text { Scaling factor due to ray-tube spreading and stratification } \\ L & \text { Lagrangian } \\ N & \text { Number of steps during propagation } \\ p, P & \text { Pressure waveform during propagation } \\ q, r, t & \text { Intermediate pressure waveforms } \\ \text { Subscripts } & \\ n & \text { Propagation iteration counter }\end{array}$

\section{Introduction and Motivation}

Development of novel, efficient and reliable methods to design supersonic aircraft for the purpose of sonic boom mitigation remains one of the most important steps in the conceptual and preliminary design stages. Since the 1960 's, researchers ${ }^{1,2,3}$ realized the importance of aircraft shaping in reducing the sonic boom impact. The Shaped Sonic Boom Demonstrator (SSBD) ${ }^{4}$ program verified, via flight testing, that aircraft shaping is an effective strategy for changing the boom signature on the ground. Since this ground-breaking study, there have been persistent efforts directed towards achieving better designs (For eg. Quiet Spike ${ }^{\mathrm{TM}}$ Ref. [5]) to reduce the boom footprint.

Sonic boom analysis can be divided into different fidelity levels. Traditionally, analyses based on equivalent-area concepts have been grouped into low-to-medium fidelity methods because equivalentarea concepts are direct off-shoots of the linearized perturbation theory. Analysis methods based on Computational Fluid Dynamics (CFD) are considered to be high fidelity.

While there have been studies ${ }^{6}$ that show some promise towards achieving sonic boom mitigation without the use of sensitivity information, the design approaches based on sensitivity of pressure distributions to the aircraft shape offer an effective and theoretically sound way to reduce the adverse impact of sonic boom. Large scale CFD simulations are widely used for aerodynamic analyses of flows around aircraft configurations. Adjoint-based methods provide effective tools to compute sensitivities of various aerodynamic quantities to many shape design parameters. Several studies ${ }^{7,8}$ demonstrated capabilities of adjoint-based methods to optimize near-field sonic boom waveforms.

In the current study, the aerodynamic analysis is performed with a CFD code, FUN3D, developed at NASA Langley. This code provides discretely consistent adjoint capabilities for sensitivity analysis. For many years, FUN3D has been used to perform adjoint-based mesh adaptation ${ }^{9,10,11,12}$ and design optimization, ${ }^{13,14}$ including optimization of the near-field sonic-boom waveforms, which represent the current state of the art in sonic boom mitigation. In the present paper, we are looking beyond the current state-of-the-art approaches to directly optimize ground-based sonic boom metrics rather than continue working on near-field target matching. With this motivation, a high fidelity design approach that included propagation to the ground within the design optimization process was proposed earlier. ${ }^{15}$ This approach formally coupled a CFD adjoint methodology with a boom propagation adjoint method. The objective was to minimize the difference between the computed ground signature and a target ground signature.

While optimizing ground signatures is a step closer to the ultimate design intent than optimizing near-field pressure waveforms, it perhaps is still limited in applicability. The primary reason for this is that specifying a suitable ground target for the chosen geometry parameterization scheme is challenging. Merely smoothing a baseline ground signature or specifying a smooth sine-wave like signature can lead to situations where the optimizer may not be able to reach this prescribed target. Moreover, the minimization of the sum of squared errors between the target and design signatures does not necessarily translate to reduced loudness values. This is because loudness values depend on the frequency content of the ground signature, and cannot be captured by the sum of squared errors. To overcome these problems, this paper suggests to use a metric based on sonic boom ground signature loudness as the optimization objective. This guarantees that the signature obtained for the optimized design will have a loudness value lower than the signature of the baseline configuration. The methodology described in this study does not necessarily replace current state-of-the-art approaches; it complements them by providing a useful tool for additional shape optimization.

The main goals of this paper are:

- To formulate the loudness adjoint problem

- Predict the sensitivities of a loudness (A-weighted loudness) objective w.r.t. selected design variables 
- Couple boom adjoint method with an adjoint CFD solver

- Demonstrate the functionality using an aircraft shape optimization exercise

\section{Mathematics of Boom Adjoint}

This section presents the mathematics of boom adjoint methodology. The primal problem refers to the augmented Burgers' propagation ${ }^{16}$ and is given in Equation 1.

$$
\frac{\partial P}{\partial \sigma}=P \frac{\partial P}{\partial \tau}+\frac{1}{\Gamma} \frac{\partial^{2} P}{\partial \tau^{2}}+\Sigma_{\nu} \frac{C_{\nu} \frac{\partial^{2}}{\partial \tau^{2}}}{1+\theta_{\nu} \frac{\partial}{\partial \tau}} P-\frac{1}{2 G} \frac{\partial G}{\partial \sigma} P+\frac{1}{2 \rho_{0} c_{0}} \frac{\partial\left(\rho_{0} c_{0}\right)}{\partial \sigma} P
$$

An operator splitting scheme ${ }^{16,17}$ is used to solve a set of five equations under the assumption that if the time step is small, the error induced by splitting is small. Equation 2 represents the effect of first relaxation and scaling due to ray tube area spreading and stratification. The matrices included in these equations are provided in the Appendix. Based on the discretization scheme used, the matrices are tridiagonal; hence the Thomas algorithm ${ }^{18}$ may be used to solve the system efficiently. Since there are two relaxation phenomena corresponding to Oxygen and Nitrogen, Equations 2 and 3 are each solved using their respective values for $C_{\nu}$ and $\theta_{\nu}$.

$$
\begin{gathered}
A^{n} q_{n}=k_{n} B^{n} p_{n-1} \\
A_{2}^{n} r_{n}=B_{2}^{n} q_{n}
\end{gathered}
$$

For the absorption equation, a Crank-Nicholson scheme is used for advancing the pressure in time. Using this discretization scheme, the absorption phenomenon also transforms into a tridiagonal matrix problem as given in Equation 4, which is solved to obtain $t_{n}$.

$$
A_{3}^{n} t_{n}=B_{3}^{n} r_{n}
$$

The non-linear equation is solved using the Poisson solution and is dependent on the solution from the absorption equation as given in Equation 5. In this equation, $t_{n}$ is a function with two arguments, the propagation distance $\left(\sigma_{n}\right)$ and the time coordinate; $t_{n}$ can be thought of as a matrix such that $t_{n, i}$ represents the $\sigma_{n}^{t h}$ row and $\tau_{i}^{t h}$ column. This retarded time equation is solved via re-interpolation as shown in Equation 6, where $\tau^{\prime}$ is the retarded coordinate given by $\tau_{n, i}^{\prime}=\tau_{i}-t_{n, i} \Delta \sigma_{n}, \Delta \sigma_{n}=\sigma_{n}-\sigma_{n-1}$, and $j$ is an index such that $\tau_{n, i-1}^{\prime}<\tau_{j}<\tau_{n, i}^{\prime}$. Expanding the terms results in the discretized equation for the non-linear part of the Burgers' equation primal problem as given in Equation 7.

$$
\begin{gathered}
p\left(\sigma_{n}, \tau_{i}\right)=t_{n}\left(\sigma_{n}, \tau_{i}+t_{n, i} \Delta \sigma_{n}\right) \\
p_{n, j}=t_{n, i-1}+\frac{t_{n, i}-t_{n, i-1}}{\tau_{n, i}^{\prime}-\tau_{n, i-1}^{\prime}}\left(\tau_{j}-\tau_{n, i-1}^{\prime}\right) \\
p_{n, j}=t_{n, i-1}+\frac{t_{n, i}-t_{n, i-1}}{\Delta \tau-\left(t_{n, i}-t_{n, i-1}\right) \Delta \sigma_{n}}\left[\tau_{j}-\tau_{i-1}+t_{n, i-1} \Delta \sigma_{n}\right]=f_{n, j}
\end{gathered}
$$

The ray tube spreading and atmospheric stratification are simply scaling terms - these are included in the $k$ factor in Equation 2. For the solution of the augmented Burgers' equation, Equations 2, 3, 4 and 7 are solved repeatedly, in that order, for $n=1 \ldots N$ time steps and at each stage the pressure is updated, while also successively updating intermediate values: $r, q$, and $t$.

\section{Adjoint}

The discrete adjoint equations are derived in this section based on a similar implementation given by Nielsen et.al ${ }^{13}$ and Rallabhandi. ${ }^{15}$ If $\mathrm{D}$ is the vector of design variables and $I_{n}$ is the objective function, then the Lagrangian corresponding to this objective may be written as in Equation 8. Taking the derivative of the Lagrangian with respect to D results in Equation 9, where it has been assumed that the 
objective does not depend explicitly on the intermediate pressure vectors $r, q$, and $t$. Furthermore, the matrices themselves do not depend on the initial pressure profile.

$$
\begin{gathered}
L(p, q, r, t, D)=I_{N}\left(p_{N}, D\right)+\sum_{n=2}^{N} \gamma_{0, n}^{T}\left[A^{n} q_{n}-k_{n} B^{n} p_{n-1}\right] \Delta \sigma_{n}+\sum_{n=1}^{N} \gamma_{1, n}^{T}\left[A_{2}^{n} r_{n}-B^{n} q_{n}\right] \Delta \sigma_{n} \\
+\sum_{n=1}^{N} \beta_{n}^{T}\left[A_{3}^{n} t_{n}-B_{3}^{n} r_{n}\right] \Delta \sigma_{n}+\sum_{n=1}^{N} \lambda_{n}^{T}\left[p_{n}-f^{n}\left(t_{n}, D\right)\right] \Delta \sigma_{n}+\gamma_{0,1}^{T}\left[A^{1} q_{1}-k_{1} B^{1} D\right] \Delta \sigma_{n} \\
\frac{d L}{d D}=\left[\frac{\partial I_{N}}{\partial D}+\frac{\partial I_{N}}{\partial p_{N}} \frac{\partial p_{N}}{\partial D}\right]+\sum_{n=2}^{N} \gamma_{0, n}^{T}\left[A^{n} \frac{\partial q_{n}}{\partial D}-k_{n} B^{n} \frac{\partial p_{n-1}}{\partial D}\right] \Delta \sigma_{n} \\
+\sum_{n=1}^{N} \gamma_{1, n}^{T}\left[A_{2}^{n} \frac{\partial r_{n}}{\partial D}-B_{2}^{n} \frac{\partial q_{n}}{\partial D}\right] \Delta \sigma_{n}+\sum_{n=1}^{N} \beta_{n}^{T}\left[A_{3}^{n} \frac{\partial t_{n}}{\partial D}-B_{3}^{n} \frac{\partial r_{n}}{\partial D}\right] \Delta \sigma_{n} \\
+\sum_{n=1}^{N} \lambda_{n}^{T}\left[\frac{\partial p_{n}}{\partial D}-\frac{\partial f_{j}^{n}}{\partial t_{n}} \frac{\partial t_{n}}{\partial D}\right] \Delta \sigma_{n}+\gamma_{0,1}^{T}\left[A^{1} \frac{\partial q_{1}}{\partial D}-k^{1} B^{1}\right] \Delta \sigma_{n}
\end{gathered}
$$

Collecting the $\frac{\partial p_{n}}{\partial D}, \frac{\partial t_{n}}{\partial D}, \frac{\partial r_{n}}{\partial D}$ and $\frac{\partial q_{n}}{\partial D}$ terms from Equation 9 and equating them to zero results in a system of four adjoint equations that is solved iteratively backwards in time. Collecting all the $\frac{\partial p_{n}}{\partial D}$ terms, and simplifying yields Equation 10. Similarly, collecting the $\frac{\partial t_{n}}{\partial D}, \frac{\partial r_{n}}{\partial D}$, and $\frac{\partial q_{n}}{\partial D}$ terms, we have Equations 11, 12 and 13, respectively. The adjoint solution process involves solving Equations 10, 11, 12, and 13 iteratively. Equation 10 is solved initially by assuming $\gamma_{0, N+1}=0$ since there are no "N+1" terms in our primal propagation problem. The intermediate adjoints are successively updated and solved. The primal problem is solved first, and relevant pressure vectors are stored for use in the adjoint process.

$$
\begin{gathered}
\lambda_{n}^{T}=-\frac{\partial I_{N}}{\partial p_{N}}+\gamma_{0, n+1}^{T} k_{n+1} B^{n+1} \\
\beta_{n}^{T} A_{3}^{n}=\lambda_{n}^{T} \frac{\partial f_{j}^{n}}{\partial t_{n}} \\
\gamma_{1, n}^{T} A_{2}^{n}=\beta_{n}^{T} B_{3}^{n} \\
\gamma_{0, n}^{T} A^{n}=\gamma_{1, n}^{T} B_{2}^{n}
\end{gathered}
$$

\section{Problem Setup}

The ultimate objective is the reduction of the sonic boom impact at the ground level through the use of A-weighted loudness. As mentioned in the introduction, some previous studies have looked at specifying a ground $\operatorname{target}^{15}$ as well as an off-body $\operatorname{target}^{8}$ to perform shape optimization. However, integrated objectives such as loudness values have significant advantages for shape optimization. The objective function used in this study is given in Equation 14, where a target A-weighted loudness value is chosen. The derivative of the cost function (Equation 15) can be used in Equation 10 to start the adjoint calculation process. The partial derivative of the A-weighted loudness with respect to the ground pressure profile is needed for this calculation. A time-domain version of the A-weighted loudness calculation procedure based on Butterworth digital filters ${ }^{19}$ was obtained from Gulfstream. This code was then numerically differentiated and modified to yield the loudness sensitivity terms in addition to the loudness values.

$$
\begin{gathered}
I_{N}=\left(d B A_{\text {design }}-d B A_{\text {target }}\right)^{2} \\
\frac{\partial I_{N}}{\partial p_{N}}=2.0\left(d B A_{\text {design }}-d B A_{\text {target }}\right) \frac{\partial d B A_{\text {design }}}{\partial p_{N}}
\end{gathered}
$$

To verify that the loudness sensitivity values are correct, a complex variable version of the loudness calculation procedure has been developed. The complex variable approach ${ }^{20,21}$ has been applied in 
several other gradient verifications. The main advantage of the complex variable method is that true second-order accuracy is achieved by selecting step sizes without incurring subtractive cancellation errors typically present in real-valued finite differences. Table 1 shows the comparison of the loudness sensitivity values computed using the analytically differentiated loudness code and the complex version of the code for arbitrarily selected indices in the ground signature. Here, it can be seen that the values match to up to 14 decimal places thus verifying that the loudness sensitivity values are accurate for our purposes.

Table 1. Comparison of the Direct and Complex Variable Loudness Gradients

\begin{tabular}{ccc}
\hline \hline Grid Point & Direct Gradient & Complex Variable Gradient \\
\hline 55 & -1.7810273118252038 & $-1.78102731182520 \underline{47}$ \\
328 & -0.005301921655030 & $-0.0053019216550 \underline{29}$ \\
800 & -0.002079879894158 & -0.002079879894158 \\
855 & 0.000376554607869 & 0.000376554607869 \\
\hline \hline
\end{tabular}

Other cost functions can be included into the optimization framework as well. These could either be matching a target ground signature as in Equation 16 or a combination of A-weighted loudness and target ground signature as in Equation 17 or just the A-weighted loudness as in Equation 18 or other appropriate combinations of under-track and off-track signature metrics. Cost functionals that attempt to match a target reversed equivalent area ${ }^{22}$ have also been used, but are not presented in this study. For each chosen cost functional, Equation 10 needs to be updated to obtain the adjoint solutions.

$$
\begin{gathered}
I_{2, N}=\sum_{i=1}^{M}\left[p_{N}^{i}-p_{t}^{i}\right]^{2} \\
I_{3, N}=\left(d B A_{\text {design }}-d B A_{\text {target }}\right)^{2}+\sum_{i=1}^{M}\left[p_{N}^{i}-p_{t}^{i}\right]^{2} \\
I_{4, N}=d B A_{\text {design }}
\end{gathered}
$$

\section{Gradient Calculation}

For adjoint solutions satisfying Equations 10-13, the only remaining term is the last term shown in Equation 19. After the adjoint equations are solved, the last solution of Equation 13 is multiplied with the scaling factor and the tridiagonal matrix of the first relaxation process to generate the gradient values needed for optimization.

$$
\frac{d L}{d D}=-\gamma_{0,1}^{T} k_{1} B^{1} \Delta \sigma_{1}
$$

\section{Verification of Adjoint Sensitivities}

Boom propagation starts by obtaining off-body pressure distributions of the baseline concept using CFD. The propagation process discretizes these CFD off-body waveforms into desired uniform spacing grids and extrapolates these towards the ground. During this extrapolation, intermediate waveforms are stored for use in the adjoint method. The adjoint method is run using the cost function described in Equation 14. To verify the accuracy of the adjoint implementation, comparisons are made with gradients generated through the use of a complex variable approach. The propagation process is modified to work with complex variables and the derivatives of the loudness with respect to the design variables (off-body pressure distribution in this case) are calculated using an imaginary step size of $10^{-20}$. Table 2 compares the adjoint gradients against the complex variable gradients for some arbitrary grid point locations. It is seen that the results using adjoint implementation exhibit excellent agreement with the complex-variable approach. This verifies that the gradients obtained using the adjoint approach are correct to at least eight digits of numerical precision. 
Table 2. Comparison of the Adjoint and Complex Variable Gradients for uncoupled propagation adjoint

\begin{tabular}{ccc}
\hline \hline Grid Point & Adjoint Gradient & Complex Variable Gradient \\
\hline 500 & -0.000340292805 & $-0.00034029 \underline{7025}$ \\
1000 & -0.003965588892 & $-0.00396558 \underline{4628}$ \\
2000 & 0.002360527452 & $0.002360527 \underline{\underline{300}}$ \\
5000 & 0.000162585576 & $0.00016258 \underline{\underline{973}}$ \\
8000 & 0.000002698064 & $0.000002698 \underline{950}$ \\
\hline \hline
\end{tabular}

\section{Coupled CFD/Boom Adjoint Formulation}

The coupled adjoint formulation is essentially the same as introduced in our earlier work ${ }^{15}$ and is included here for the sake of completeness. The boom-adjoint formulation is coupled with the NASA Langley unstructured CFD solver FUN3D. ${ }^{23}$ The FUN3D software solves the compressible and incompressible forms of the steady and unsteady Euler and Reynolds-averaged Navier-Stokes equations on general static and dynamic mixed-element grid discretizations, which may optionally include overset grid topologies. The software has been used for a broad class of aerodynamic analysis and design simulations across the speed range. FUN3D also offers a discretely-consistent adjoint implementation that has been used to perform mathematically-rigorous design optimization, error estimation, and formal mesh adaptation for complex geometries and flow-fields in massively parallel computing environments. ${ }^{13,24}$ These applications include accurate analysis and design optimization of aircraft concepts aimed at sonic boom mitigation. ${ }^{11,10}$ Such simulations have traditionally relied on objective functions posed in the near-field within 20 body lengths of the vehicle, ultimately yielding an indirect approach which fails to formally address the pressure signature on the ground. However, the adjoint approach for the propagation methodology developed here offers an exciting opportunity to formally couple existing near-field CFD analysis and design capabilities with the methodology used to predict ground-based metrics. Finally, it should be noted that FUN3D also offers a discretely-consistent forward mode of differentiation. A scripting procedure ${ }^{25}$ can be used to automatically convert the baseline source code to a complex-variable formulation as described above. In this manner, sensitivities of all FUN3D outputs with respect to any input parameter may be easily evaluated.

The coupled formulation is described from the perspective of the CFD solver. In this approach, the interface between FUN3D and boom propagation takes the form of a one-dimensional pressure distribution $p_{0}$ evaluated at a fixed distance from the aircraft in the near-field CFD mesh. The CFD solution determined on the unstructured mesh is used to construct this pressure distribution, which serves as the input for the boom analysis problem. Given $p_{0}$, the forward mode of boom analysis evaluates the cost function $l_{N}$. The adjoint mode then determines the sensitivity of the cost function to $p_{0}$, which is a horizontal vector denoted $d l_{N} / d p_{0}$.

The relationship between the near-field pressure signature and the CFD solution is described as

$$
p_{0}=\mathbf{T}(\mathbf{Q}, \mathbf{X}),
$$

where the vectors $\mathbf{Q}$ and $\mathbf{X}$ represent the CFD solution and mesh, respectively; and $\mathbf{T}$ is a transformation mapping the CFD solution to the desired pressure distribution $p_{0}$. The Lagrangian for the coupled formulation is defined as

$$
\mathbf{L}\left(\mathbf{D}, \mathbf{Q}, \mathbf{X}, \boldsymbol{\Lambda}_{f}, \boldsymbol{\Lambda}_{g}, \boldsymbol{\Lambda}_{b}\right)=l_{N}+\left[\boldsymbol{\Lambda}_{g}\right]^{T} \mathbf{G}+\left[\boldsymbol{\Lambda}_{f}\right]^{T} \mathbf{R}+\left[\boldsymbol{\Lambda}_{b}\right]^{T}\left(p_{0}-\mathbf{T}\right) .
$$

Here, $\boldsymbol{\Lambda}_{f}$ and $\boldsymbol{\Lambda}_{g}$ are adjoint variables corresponding to the discrete CFD flow equations $\mathbf{R}(\mathbf{Q}, \mathbf{X}, \mathbf{D})=0$ and CFD grid equations $\mathbf{G}(\mathbf{X}, \mathbf{D})=0$, respectively; $\boldsymbol{\Lambda}_{b}$ is a vector of adjoint variables associated with the boom interface given by Equation 20; and $\mathbf{D}$ is a vector of design variables. In the current study, the design variables consist of geometric parameters defining the discrete surface grid for the aircraft.

Differentiating the Lagrangian with respect to $\mathbf{D}$ and equating the coefficients of $\partial p_{0} / \partial \mathbf{D}, \partial \mathbf{X} / \partial \mathbf{D}$, and $\partial \mathbf{Q} / \partial \mathbf{D}$ to zero yields the following system of adjoint equations:

$$
\begin{aligned}
& {\left[\frac{d l_{N}}{d p_{0}}\right]^{T}+\boldsymbol{\Lambda}_{b}=0,} \\
& {\left[\frac{\partial \mathbf{R}}{\partial \mathbf{Q}}\right]^{T} \boldsymbol{\Lambda}_{f}-\left[\frac{\partial \mathbf{T}}{\partial \mathbf{Q}}\right]^{T} \boldsymbol{\Lambda}_{b}=0,} \\
& {\left[\frac{\partial \mathbf{G}}{\partial \mathbf{X}}\right]^{T} \boldsymbol{\Lambda}_{g}+\left[\frac{\partial \mathbf{R}}{\partial \mathbf{X}}\right]^{T} \boldsymbol{\Lambda}_{f}-\left[\frac{\partial \mathbf{T}}{\partial \mathbf{X}}\right]^{T} \boldsymbol{\Lambda}_{b}=0 .}
\end{aligned}
$$


Recall that the vector $d l_{N} / d p_{0}$ is computed using the adjoint mode of boom analysis as described in Sections II and III. Assuming that the adjoint variables satisfy Equations 22 and that the transformation $\mathbf{T}$ given by Equation 20 does not explicitly depend on $\mathbf{D}$, the desired sensitivity derivatives of the ground signature with respect to the aircraft geometry are then calculated as follows:

$$
\frac{\partial \mathbf{L}}{\partial \mathbf{D}}=\left[\boldsymbol{\Lambda}_{g}\right]^{T} \frac{\partial \mathbf{G}}{\partial \mathbf{D}}+\left[\boldsymbol{\Lambda}_{f}\right]^{T} \frac{\partial \mathbf{R}}{\partial \mathbf{D}} .
$$

Note that the computational cost associated with the solution of Equations 22 is similar to that of their traditional forward-mode counterparts, and the cost required to evaluate Equation 23 is trivial. In this manner, the approach outlined here ultimately enables a discretely consistent sensitivity analysis to be performed for the coupled system at the cost of a single forward-mode analysis, even for very large numbers of design variables.

\section{Initial Mesh and Geometry Parameterization}

The adjoint formulation is applied over a supersonic concept shown in Figure 1. This baseline configuration is the result of earlier optimization using mixed-fidelity, ${ }^{26}$ reversed-equivalent-area ${ }^{22}$ methods. The initial mesh for this concept was generated using VGRID, ${ }^{27}$ SSGRID $^{28}$ and is shown in Figure 2. This grid generation approach is a heuristic technique to align the mesh topology a priori with the expected primary off-body shock structures. A more rigorous adjoint-based approach to mesh adaptation for such problems is described in literature. ${ }^{10}$ The CFD grid utilizes a plane of symmetry along the centerline and contains 4,163,500 nodes and 23,991,137 tetrahedral elements. The surface mesh for the aircraft has been parameterized using the packages MASSOUD ${ }^{29}$ and BANDAIDS. ${ }^{30}$ These methodologies are based on free-form deformation techniques which provide a compact set of design variables describing changes to a discrete surface mesh. Both approaches provide the analytic sensitivities required by the discrete adjoint formulation of the near-field CFD problem. MASSOUD ${ }^{29}$ is designed for use with common aircraft-centric geometries and provides a set of intuitive design variables such as thickness, camber, twist, shear, and planform parameters. For the current test, this approach has been used to parameterize the main wing, and horizontal tail surfaces. BANDAIDS ${ }^{30}$ was used to parameterize and modify the nose section of the baseline. This technique is more appropriate for general surface topologies and provides a set of design variables describing general displacements normal to a surface. For simplicity, the other components are not allowed to vary and the intersections between aircraft components are held fixed, although this is not a requirement of the formulation. A total of 562 design variables were used to parameterize the above-mentioned components of the aircraft concept, but only 373 of them are active during the optimization.

Similar to the previous verification cases, the coupled-adjoint sensitivities are compared against complex sensitivities for the coupled problem. It has been noted that the sensitivity values match well up to 8 digits of numerical precision. This is the same accuracy achieved from the propagation adjoint alone; therefore the coupled problem accuracy match is not expected to be better. Since sensitivities match for several decimal digits, the adjoint sensitivities can be used effectively during numerical optimization.

\section{Computational Results}

This section presents the results of the coupled-adjoint formulation for complete configurations in supersonic flow with free-stream Mach numnber of 1.6 and angle of attack of 0.6 degrees. The objective is to reduce the A-weighted loudness of the sonic boom signatures at the ground level. Viscous effects are likely to be small for these configurations when the primary objective is sonic boom; therefore we limit ourselves to Euler equations for this study. The calculations were carried out on 16 nodes of Altix ICE 8400 cluster at NASA Langley Research Center. Each node contains dual processor, hex core 3.07 Ghz Westmere CPUs, making it 12 cores per node and 24 GBs of memory (or 2 GBs per core).

\section{A. Flow-Through Nacelle}

Optimization was first attempted using a flow-through engine setting where the nacelles are almostconstant area ducts. To simplify the problem and reduce the number of design variables, the shape changes are limited to wing, horizontal-tail and the nose sections of the fuselage. Figure 3 shows the ground signature of the baseline along with the signature obtained when the optimizer stops. The optimizer successfully smooths out the front portion of the signature, and attempts to break up the stronger aft shocks. The A-weighted loudness reduces from a value of $64.74 \mathrm{dBA}$ for the baseline to a value of $62.49 \mathrm{dBA}$. Table 3 provides other metrics corresponding to these ground signatures. The total 


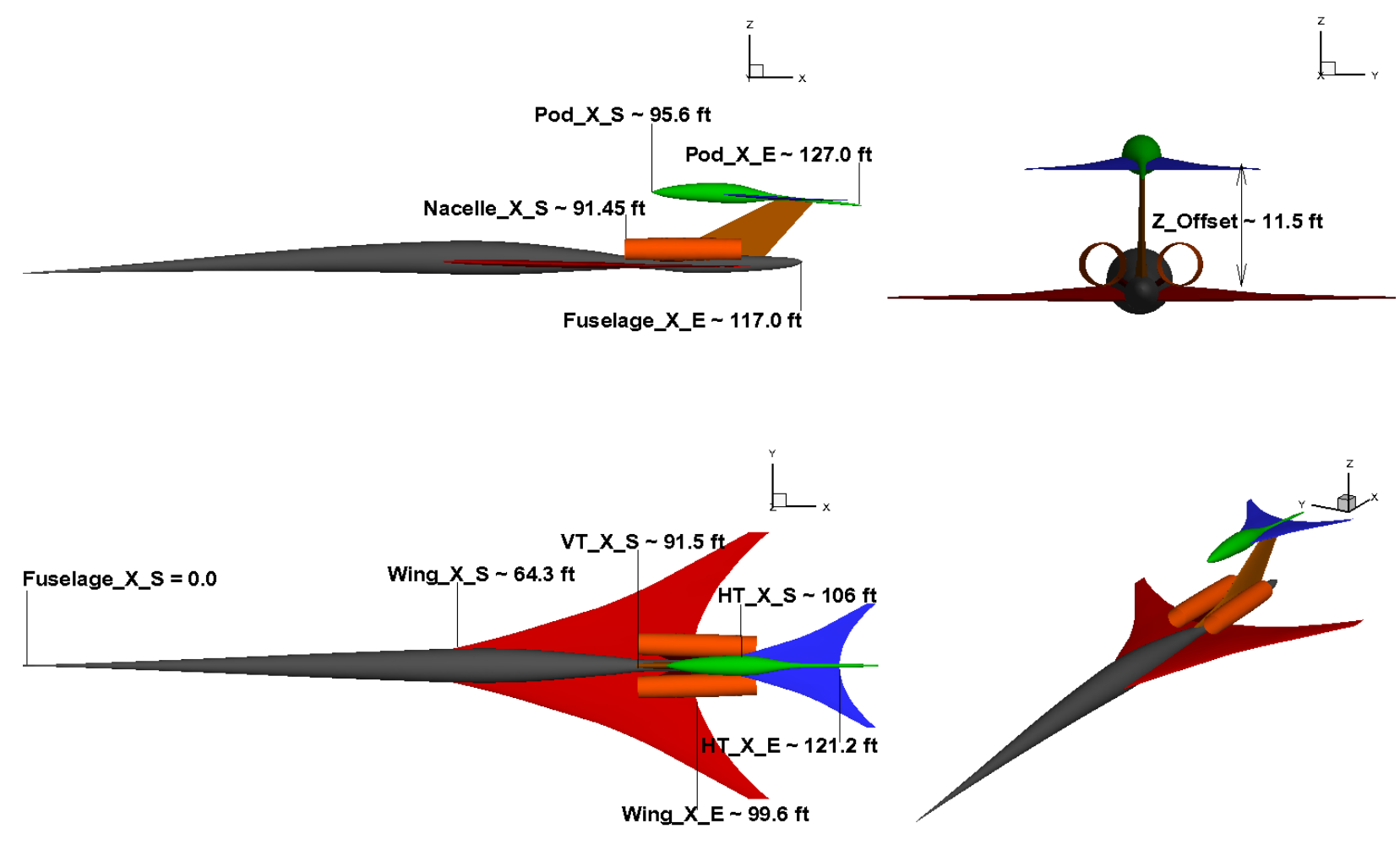

Figure 1. Orthogonal projections of the Baseline configuration

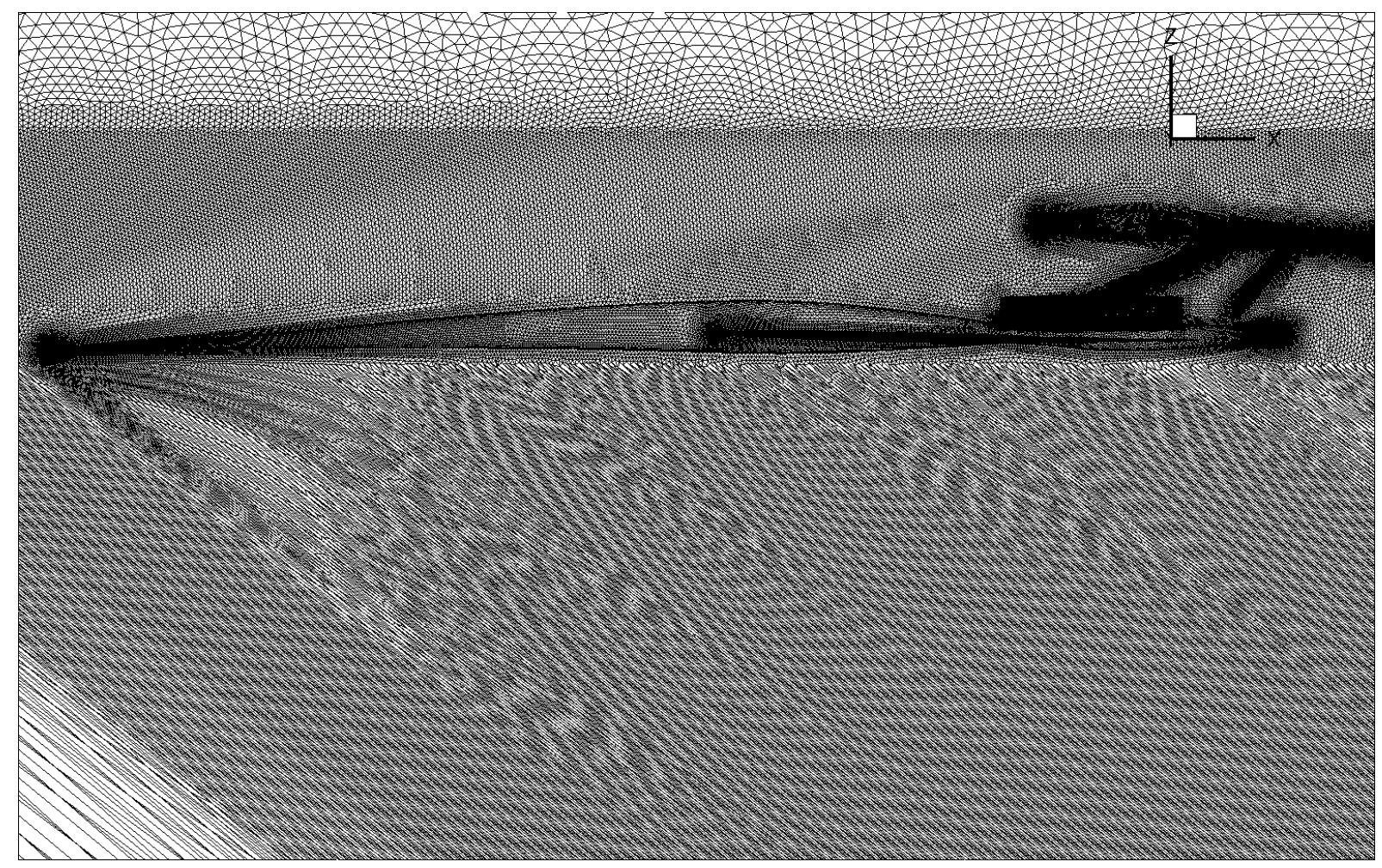

Figure 2. CFD stretched grid 
perceived loudness drops by around $1.5 \mathrm{~dB}$ compared to the baseline. The perceived loudness contribution from the front part of the signature drops by around $3 \mathrm{~dB}$, while the reduction on the aft part is much smaller. The optimizer stops before it can make progress on the backend because a few design variable bounds are hit and hence further optimization is not allowed. The design variable bounds were increased to allow the optimizer to explore more design space; however the optimizer terminated at a location that has higher cost functional than when the bounds were tighter. Such increases in the cost functional are the result of the presence of multiple local minima; extension of the design space leads the optimizer to choose a different path to convergence before settling on another local minimum. Different optimization packages such as NPSOL, ${ }^{31}$ PORT, ${ }^{32}$ and KSOPT $^{33}$ were tried during the course of this study and their convergence behavior was somewhat similar. For the problem at hand, NPSOL seemed to make aggressive steps and reaches the optimum in the least amount of time; hence NPSOL was used in all the results presented in this study.

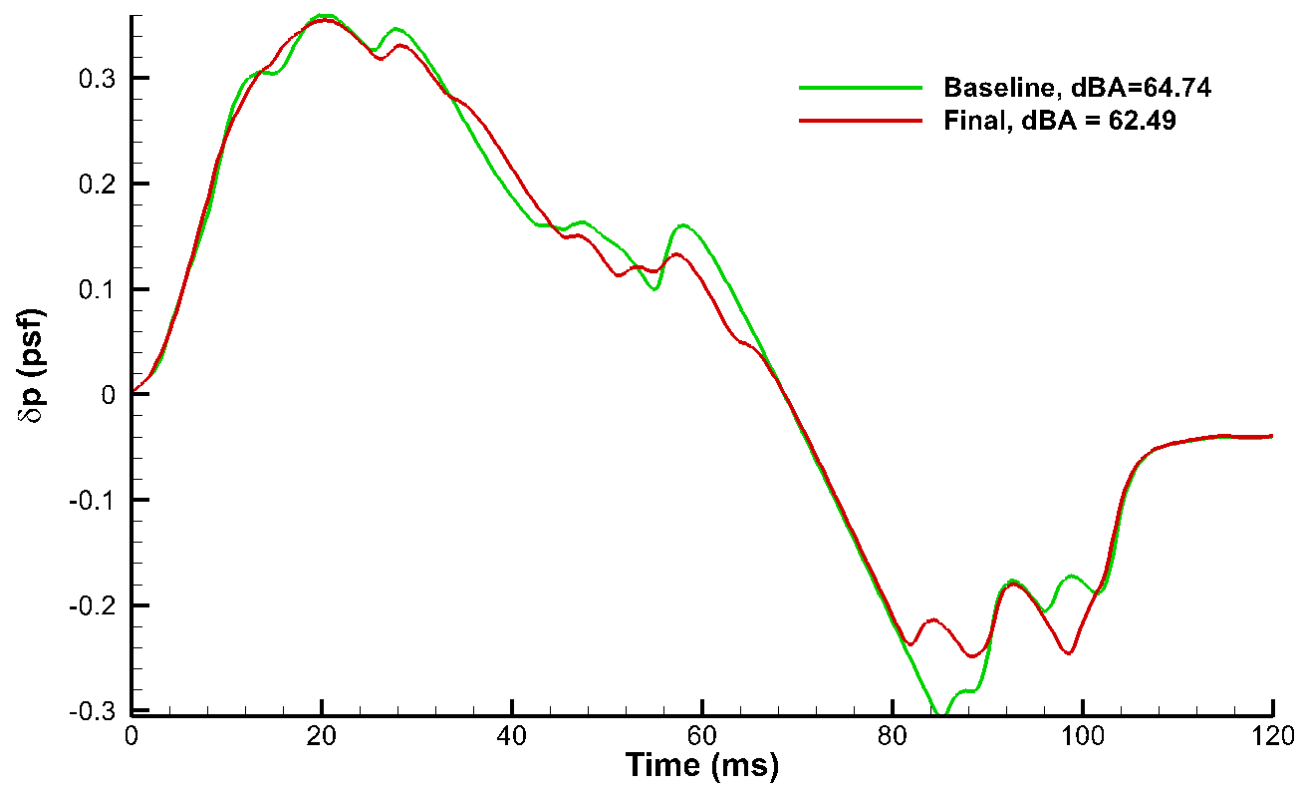

Figure 3. Baseline and Final Ground Signatures

Table 3. Comparison of metrics corresponding to the Ground Signatures

\begin{tabular}{cccccc}
\hline \hline Case & dBA & PLdB Front & PLdB Aft & PLdB Total & L/D \\
\hline Baseline & 64.74 & 76.2 & 76.7 & 79.0 & 7.41 \\
Final & 62.49 & 73.0 & 76.0 & 77.5 & 7.33 \\
\hline \hline
\end{tabular}

The optimizer makes several non-intuitive modifications to the baseline geometry in order to reduce the A-weighted loudness of the ground signature. The cross-sectional differences between the baseline and the final configuration are shown in Figure 4. Figures 4(a) and 4(b) show the changes at some cross-sections for the wing and horizontal tail respectively; the spanwise non-dimensional distance of each cross-section is also listed. The changes to the nose are too small to show the differences. The wing cross-sections after the adjoint-based optimization are quite smooth while the under-side of the horizontal tail seems to have large changes especially near the outboard sections.

Figure 5 depicts the near-field pressure distribution comparison. From this plot, one can realize that the optimizer is attempting to reposition the shocks in such a way so maximize shock cancellation through relative placement of shocks and expansion regions. The wing shock structure is redistributed so that the rear shock system is reduced. However unlike the wing and nose regions which are well separated from the other aft components, changing the horizontal tail alone in the aft portion is not enough to reposition the aft shock system. This is perhaps due to the interaction between multiple aft components (See Figure 1) that produce the aft shock-expansion system. Due to this complex interaction, the changes to the horizontal tail reduce the strengths of a couple of shocks in the near-field aft shock system; however the position of those shocks is not altered by much. The positions of the shocks and expansions are 


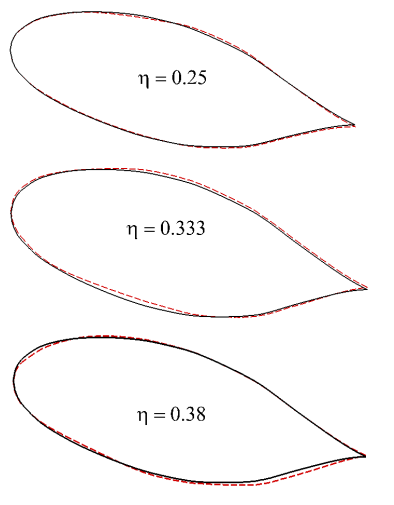

(a) Changes to the Wing
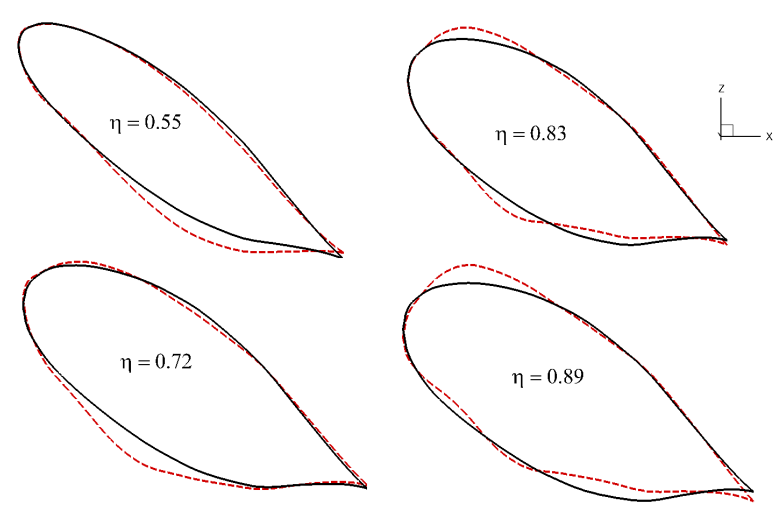

(b) Changes to the Horizontal Tail

Figure 4. Comparison of the cross-sections of the baseline and final concept

determined by other components as well, especially the pod at the intersection of the horizontal tail and the vertical tail. In order to properly change the aft shock system, several aft components need to be allowed to change their shape. The iteration history of the objective function values is plotted in Figure 6. The optimizer reduces the cost functional (indirectly the A-weighted loudness) from the baseline value of around 75 to about 42 at which point the optimizer fails to make progress and terminates.

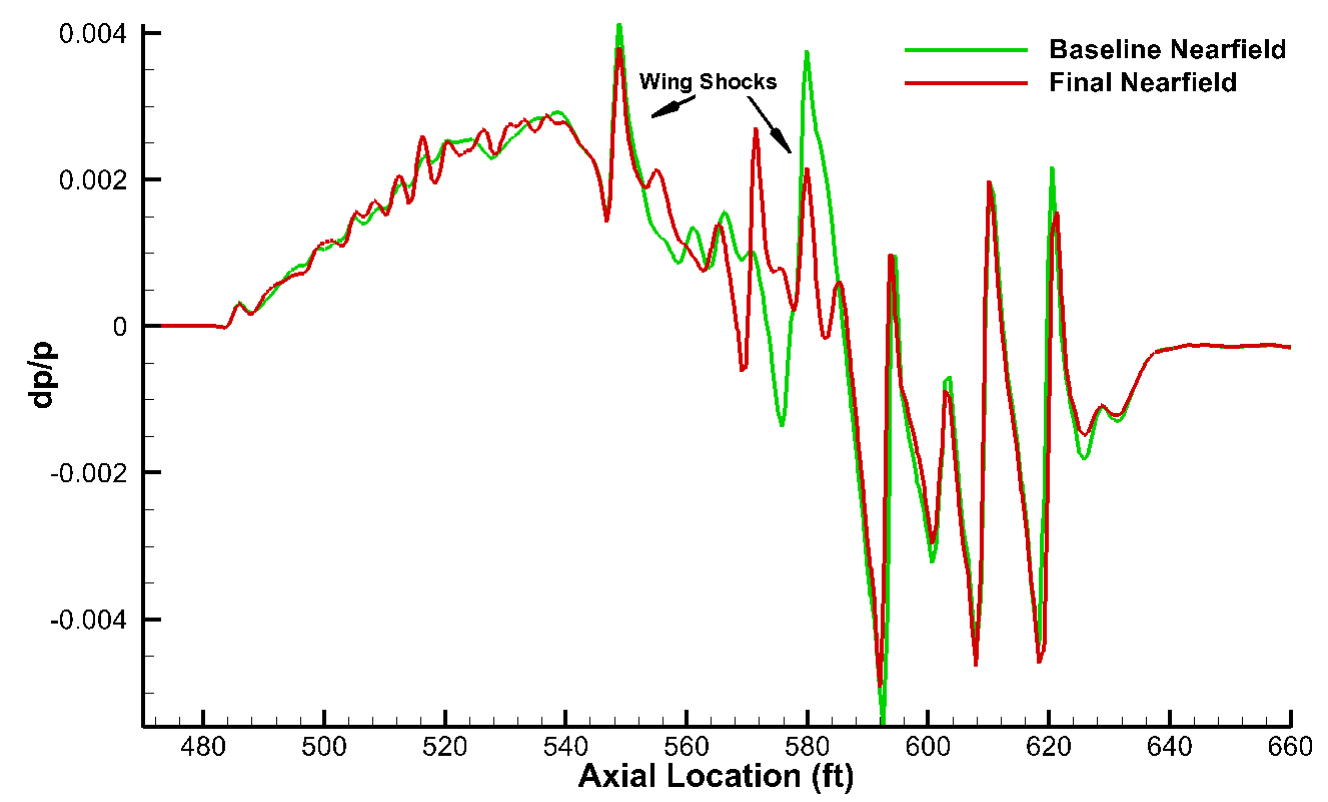

Figure 5. Baseline and Final Near-field pressure waveforms

The computational wall time for this run was about 5 hours 10 minutes with 22 flow solves and 20 adjoint solutions. The flow solver residual usually drops by six to seven orders of magnitude before declaring convergence, and the adjoint solver residual drops by twelve to thirteen orders of magnitude. The computational run times will be lower if the convergence criteria are relaxed for the flow and adjoint solvers. The breakdown of the computational time for each iteration is included in Figure 7 . The majority of the time (68\%) is spent in Flow and Adjoint solutions; however a significant portion ( 20\%) is spent in obtaining the surface sensitivities and surface mesh deformation and the remaining ( $12 \%)$ is spent during file system I/O and overhead. The parameterization packages used here currently rely on formatted file structures; this restriction is being addressed and is expected to drastically reduce the wall time associated with file I/O.

\section{Optimization with an $L / D$ constraint and larger design space}

From Figure 3, it appears as if the wing and nose have been well shaped and there have been changes to the aft portion of the signature; however only the horizontal tail was allowed to vary in the previous 
optimization run. To allow the optimizer to vary other components and further improve the signature on the back end, the pod and aft fuselage of the baseline concept were parameterized. The following cases were run:

1. The optimizer (NPSOL) was initially allowed to vary pod and aft fuselage while freezing the wing, horizontal tail and nose to the shapes obtained in the previous run. The optimizer failed to make much progress before terminating because the starting point was already near a local minima.

2. A second case was run freezing just the nose to the previously obtained optimum, but allowing wing, horizontal tail, pod and aft fuselage to vary. The optimization resulted in a final cost functional value of 57.95 , which is inferior to the result obtained when only the nose, wing and horizontal tail were allowed to vary.

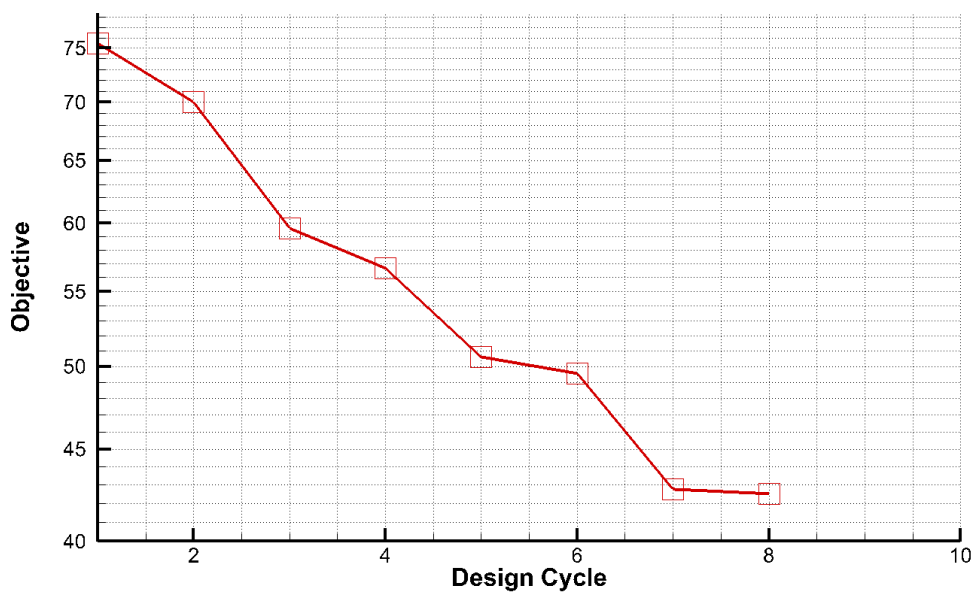

Figure 6. Iteration History of the Optimizer

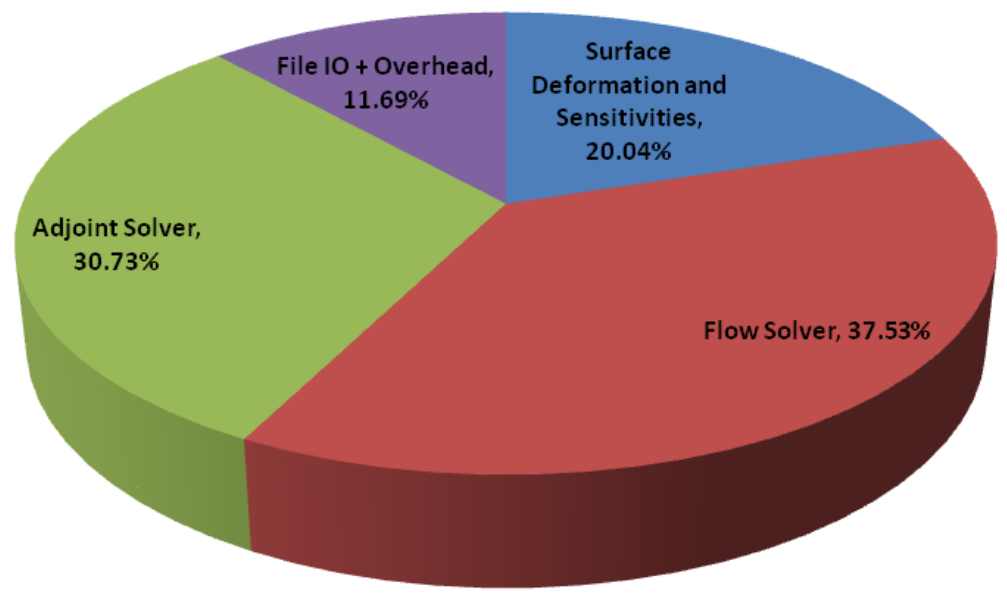

Figure 7. Computational time breakdown

3. Lastly, the optimization was run by freezing both the nose and wing to the shapes from the previous case while varying all the aft components including the horizontal tail, which is set to its baseline shape before starting the optimization run. This adds 72 design variables to the optimization problem. An explicit lift to drag constraint was also placed so that all values below 7.35 are considered infeasible by the optimizer; this constraint value was selected based on the previous case. Figure 8 shows the effect of this aft optimization where the baseline and previous ground signatures are also plotted for comparison. It is seen that allowing the aft components to change their shapes further improves the aft signature shape. Figure 9 depicts the value of the lift to drag constraint as the design changes. The loudness values corresponding to these are given in Table 4 . One interesting observation is that after aft exploration, the A-weighted loudness increases slightly when compared to the value obtained when only the horizontal tail was allowed to change in the aft portion of the aircraft; however there is a $1 \mathrm{~dB}$ drop in the perceived loudness value. In this respect, for shaped sonic booms, the correlation between the A-weighted loudness and the perceived 
loudness does not seem to be high. The optimizer is able to reduce the perceived loudness by a healthy $2.5 \mathrm{~dB}$ from the baseline concept while not significantly reducing the lift over drag ratio from the baseline value.

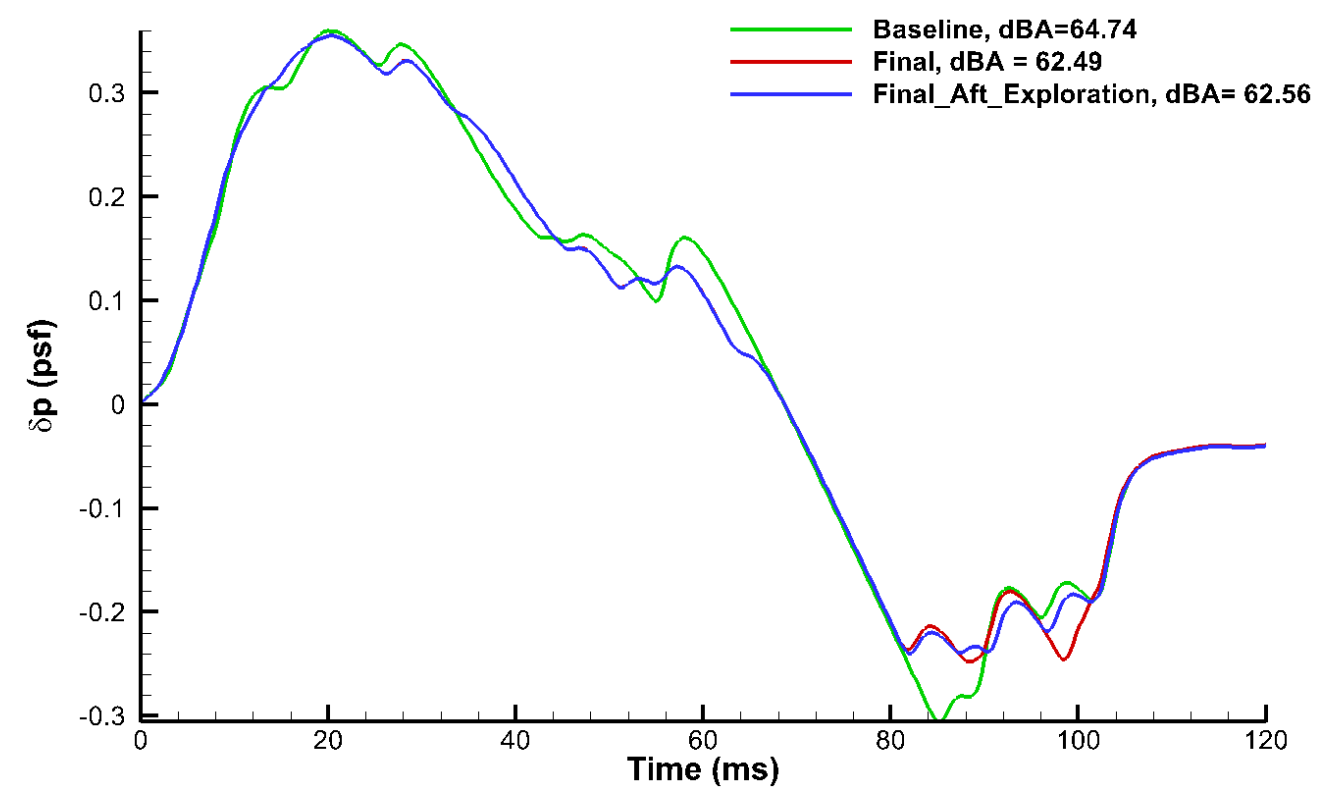

Figure 8. Baseline, Final Ground Signatures with aft component changes

The changes to the horizontal tail sections are shown in Figure 10. Compared to Figure 4(b), the sectional changes with respect to the baseline are even more subtle. There are small changes in the pod and aft fuselage sections as well. However, they are too small to be seen. Figure 11 shows the comparison of the near-field pressure waveforms after aft exploration. It can be observed that the changes in the aft portion are quite small, but have a significant impact on the aft portion of the ground signature as well as the loudness metrics.

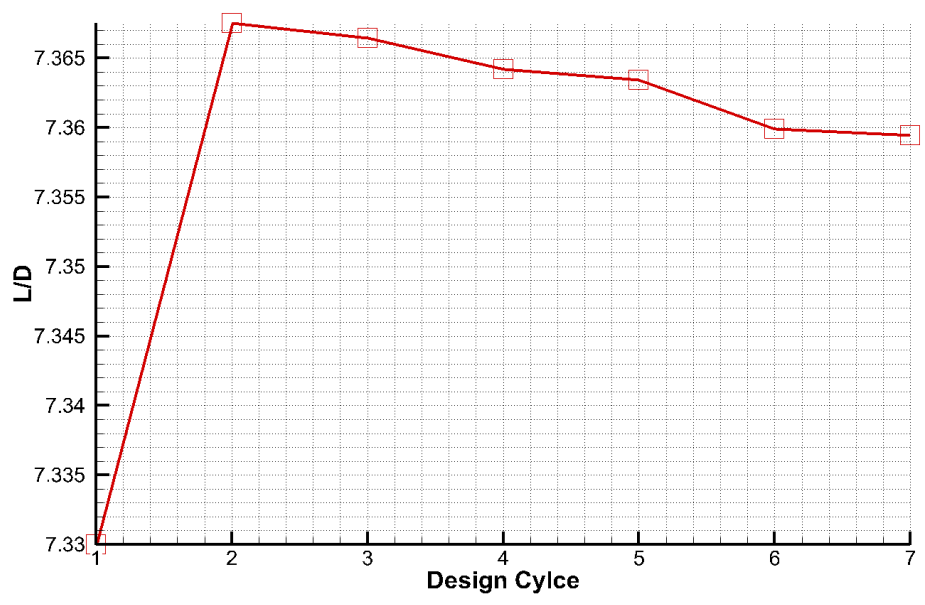

Figure 9. History of the L/D constraint during constrained aft exporation

\section{Discussion}

Several optimization runs were carried out during this study. The main lessons learned during the course of this study are detailed below.

1. During the course of the optimization, it was observed that some desired regions of the design space with large gradients were avoided because of limitations imposed by specific surface parameterizations. A remaining challenge is to choose a geometry parameterization and the corresponding bounds of the design parameters to open potentially benefecial design regions for exploration and 
Table 4. Comparison of loudness metrics of Ground Signatures

\begin{tabular}{cccccc}
\hline \hline Case & dBA & PLdB Front & PLdB Aft & PLdB Total & L/D \\
\hline Baseline & 64.74 & 76.2 & 76.7 & 79.0 & 7.41 \\
Final & 62.49 & 73.0 & 76.0 & 77.5 & 7.33 \\
Aft Exploration & 62.56 & 73.0 & 74.9 & 76.5 & 7.36 \\
\hline \hline
\end{tabular}

to ensure that the deformation of the outer mold line does not compromise the integrity of the surface meshes.

2. Choice of cost function: In the previous paper we reported on coupled adjoint formulation, ${ }^{15}$ the cost function was $\sum_{i=1}^{M}\left[p_{N}^{i}-p_{t}^{i}\right]^{2} \Delta t$. During subsequent optimization runs, the cost function was first modified to $\sum_{i=1}^{M}\left[p_{N}^{i}-p_{t}^{i}\right]^{2}$, and then to the loudness based cost function introduced in this study. The presence of $\Delta t$ in the first cost function may introduce scaling problems. Also functionals of the form of $\sum_{i=1}^{M}\left[p_{N}^{i}-p_{t}^{i}\right]^{2}$ are stiff and slow to converge.

3. The adjoint-based optimization can complement advanced conceptual design methods. In the current study, conceptual design methods initially introduced large changes in the aircraft shape, followed by adjoint-based optimization that was able to provide additional significant improvement in the cost functional under consideration. From the perspective of boom loudness, each decibel reduction is extremely crucial; a reduction of 2.5 decibels on the perceived loudness scale over an already optimized geometry is considered significant.
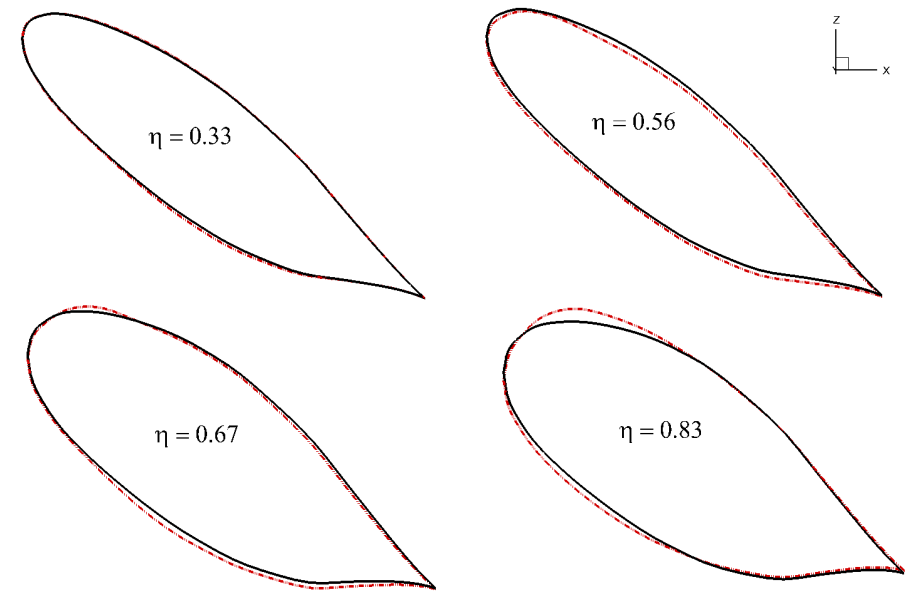

Figure 10. Comparison of the horizontal tail cross-sections after aft exploration

4. The adjoint sensitivities have been verified thoroughly with an independent complex-variable approach and have been shown to be accurate. However, most gradient-based optimizers lack the capacity to reach a global minimum especially when a noisy objective such as loudness is employed. Hence there is an urgent need to explore the use of specially designed optimizers that can tackle noisy objective functions. Alternatively, one could use an intelligent technique to obtain a starting point in the vicinity of the global minimum. Filtering techniques ${ }^{34}$ could be useful in that respect and are being explored.

5. Almost all adjoint-based shape optimization exercises in the literature for sonic boom minimization have used near-field target matching. The main reason for this is that near-field matching is an easier and more intuitive problem compared to using cost functionals on the ground. For near-field targets there is a one-to-one correspondence between the shocks and expansions in the off-body waveform and the geometry. This one-to-one correspondence is lost during propagation due to shock coalescence and other atmospheric phenomena. Thus, matching a near-field target may not be sufficient to generate an optimal ground signature. Even though optimization based on ground level objectives is more difficult and less intuitive, working directly with loudness metrics is desirable since they are the ultimate figures of merit used in acceptability. As a reference, studies ${ }^{35}$ in the 


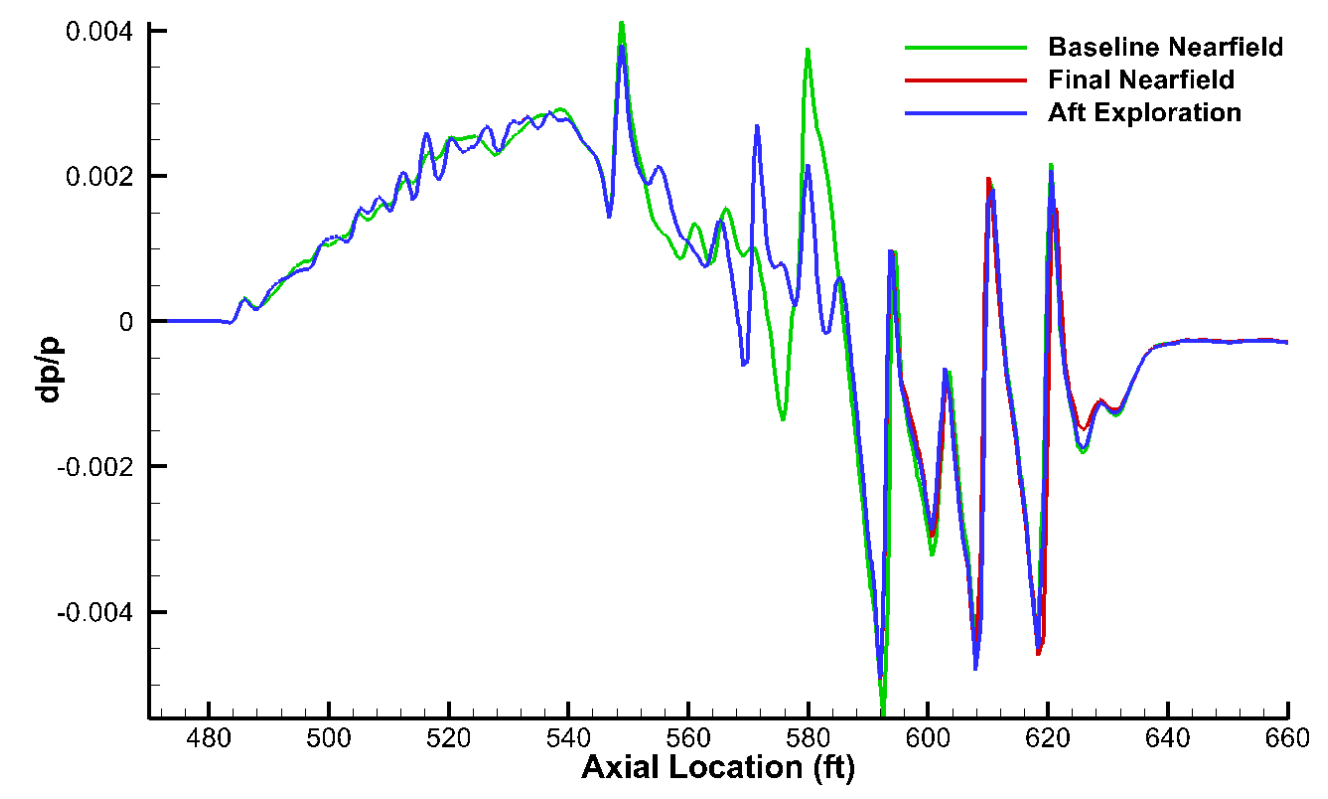

Figure 11. Comparison of the Near Fields after aft exploration

1990's had the A-weighted loudness value of 72 dBA for acceptable supersonic flight; this has since been replaced with an aggressive acceptability goal of $55 \mathrm{dBA}$. Adjoint-based methodology introduced in this paper is the first rigorous methodology that allows inclusion of integrated ground level objectives in design optimization.

6. Even though the A-weighted loudness based cost function used in this study is generally well correlated with the perceived loudness values, there are discrepancies for shaped ground signatures. In other words, for shaped ground signatures, a lower A-weighted loudness does not necessarily mean a lower perceived loudness since the weighting coefficients are different between the two loudness scales. It is desirable to consider perceived loudness or some other loudness metric that correlates well with perceived loudness in defining the cost functionals.

7. The location in the design space where the optimizer stops during a run not only depends on the design flexibility and exploration space, but also on the path the optimizer takes during the optimization algorithm. For example, allowing only a few components to change shape while freezing some components to their baseline shape may produce a better result than allowing all the components to change shape. This is because the paths taken by the optimizer in these two situations are different; thus the local minima reached are different. The same can be said if constraints are added to the problem formulation. This problem demonstrates that optimization algorithms that intelligently explore larger design spaces while overcoming local minima will need to be employed.

8. Figure 12 depicts the surface sensitivity contours of ground based cost functional with respect to the normal perturbations to the surface geometry for the baseline configuration. This figure shows that the outer sections of the wing, upper surface of the horizontal tail are relatively less important for reducing the cost functional than other areas of the wing and horizontal tail. For under-track boom metrics, as expected, the lower surface of the aircraft is much more sensitive than the upper surface; this will likely change if off-track metrics are also included in the cost functional. Figure 13 depicts the same contours when the optimizer terminates for the case without aft exploration of component changes. Since the components that were allowed to vary were just the fuselage nose, wing and horizontal tail, it is seen that the adjoint process attempts to make the design parameters associated with these components insensitive to the ground-based cost functional as evidenced by the larger fraction of green region in the sensitivity contour spectrum over portions of the fuselage nose, upper surfaces of the wing and horizontal tail as well as lower surface of the horizontal tail. However, the sensitivity contours on the bottom side of the wing seem to have increased in magnitude compared to the baseline, especially near the wing-fuselage intersection near the trailing edge, where the optimizer did not have control to make changes. These sort of plots will help in choosing appropriate design variables in the shape optimization process. These figures represent 
only a snapshot in a dynamically varying sensitivity field; as the design changes the sensitivity contours change. Future work would have to use this information to constantly update and restrict the active design variables to those that have the highest impact on the cost functional. This will help reduce the number of design variables; however the complexity of the optimzer will increase due to the additional book keeping.

While all the tools necessary for conducting a successful adjoint-based shape optimization for the purpose of mitigating sonic boom exist, further research is needed. Some of the current outstanding issues include generation of quality initial grids, appropriate geometry parameterization and setting bounds on those parameters, and improved gradient-based optimization algorithms capable of approaching the global minimum or at least overcoming shallow local minima. The adjoint method is producing reliable sensitivities for use in an optimization framework; additional work is needed on the optimization formulation. Sonic boom mitigation represents a challenging, highly integrated design problem that can be solved with the right mix of tools that have been introduced here and elsewhere in literature. The challenge is to refine the process such that each contributing analysis is robust and reliable and does not artificially constrain the optimizer in its design space exploration.

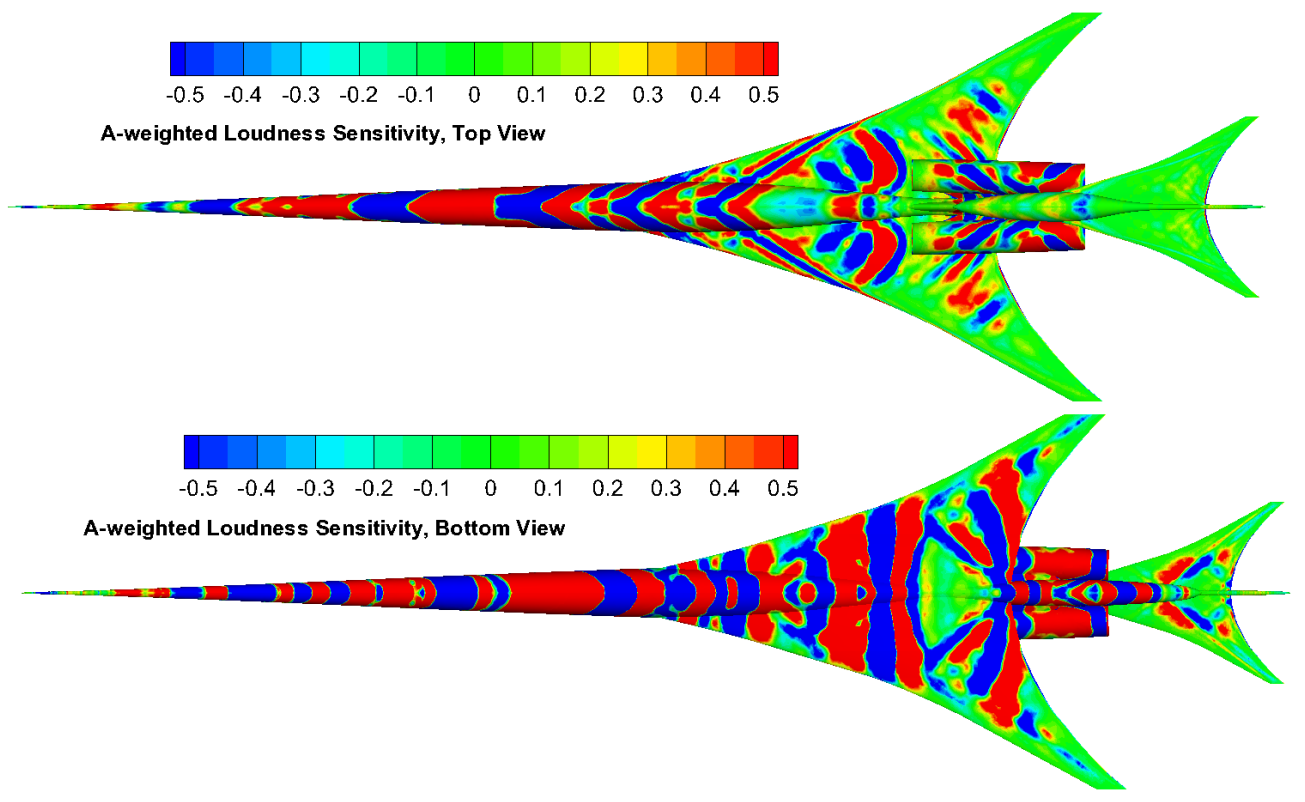

Figure 12. Surface sensitivity contours of ground based cost functional with respect to the normal perturbations to the surface geometry for the baseline configuration

\section{Conclusions}

A sonic boom ground signature loudness sensitivity method has been developed using the discrete adjoint approach and augmented Burgers' equation. The boom adjoint method has been formally coupled with a high-fidelity CFD and shape optimization environment for designing low-boom supersonic aircraft concepts. Based on the optimization results for a configuration previously optimized with other stateof-the-art design methods, significant positive changes to the ground signature are obtained by subtle non-intuitive changes to the aircraft outer mold line. The coupled adjoint-based formulation is available to include engine simulation, as well as viscous solutions if needed. Future work will attempt to utilize advanced algorithms and hybrid strategies to improve the performance of the optimizers. Additionally, adjoint mesh adaptation in conjunction with adjoint-based design will be pursued.

\section{Acknowledgments}

This work was supported by the NASA Project entitled "Sonic Boom Propagation Tools and Methods for Low Sonic Boom Design," under NASA contract number NNL08AA00B, task number NNL12AA55T. The receipt of the time-domain A-weighted loudness calculation code from Gulfstream Aerospace is gratefully acknowledged. The time and effort put in by Scott Brynildsen, Jan-Renee Carlson, Norma Farr, and Dick Campbell for geometry generation, and gridding for powered-on engine simulation in a short amount of time and Karl Geiselhart for engine boundary conditions is greatly appreciated. Help 


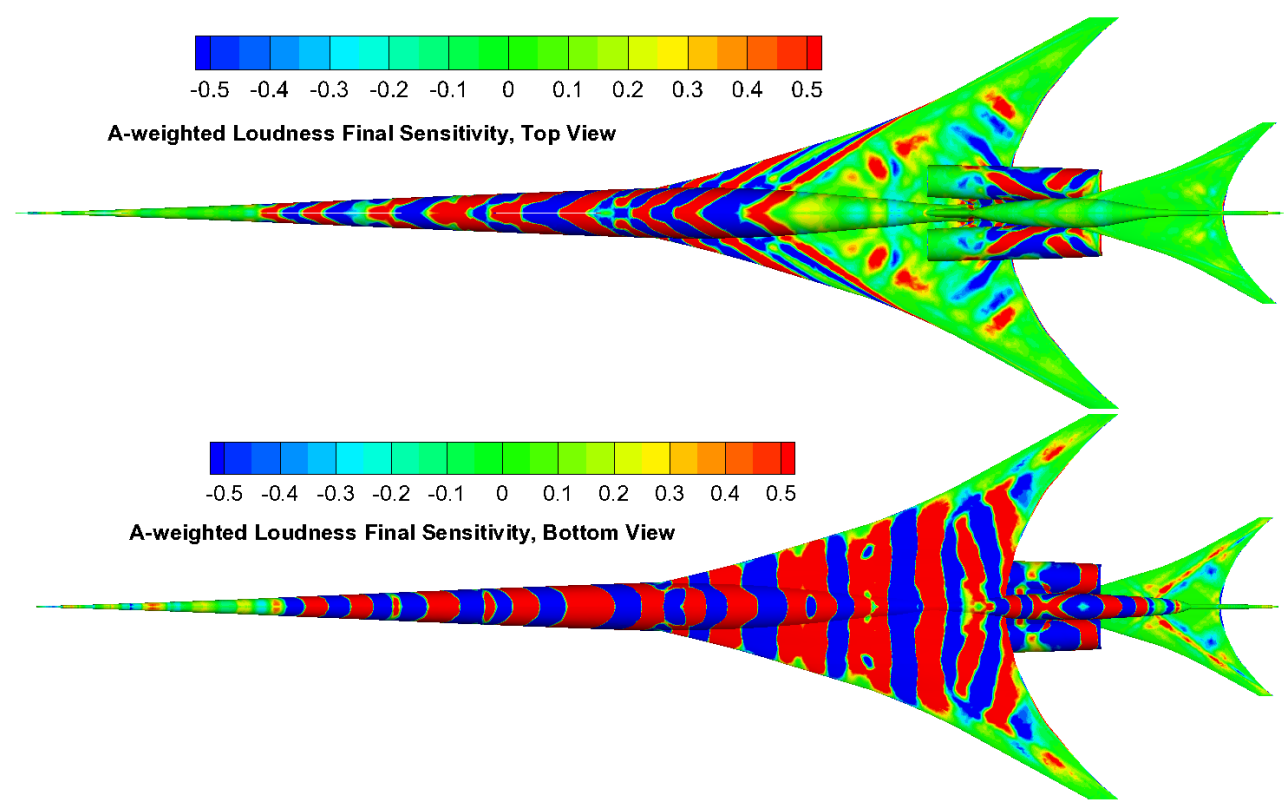

Figure 13. Surface sensitivity contours of ground based cost functional with respect to the normal perturbations to the surface geometry for the final configuration

in several forms from Lori Ozoroski, Irian Ordaz, Wu Li, Mike Park, Bill Jones and Bill Kleb is also acknowledged.

\section{References}

${ }^{1}$ George, A. R. and Seebass, R., "Sonic Boom Minimization Including Both Front and Rear Shocks," AIAA Journal, Vol. 9, No. 10, 1971, pp. 2091-2093.

${ }^{2}$ Seebass, R., "Sonic boom reduction through aircraft design and operation," AIAA Paper No. 1973-241, 1973.

${ }^{3}$ Seebass, R. and George, A., "Sonic Boom Minimization," Journal of Acoustical Society of America, Vol. 51, No. 2, 1972, pp. 686-694.

${ }^{4}$ Pawlowski, J. W., Graham, D. H., et al., "Origins and Overview of the Shaped Sonic Boom Demonstration Program," AIAA Paper 2005-5, Jan. 2005.

${ }^{5}$ Howe, D., Simmons, F., and Freund, D., "Development of the Gulfstream Quiet Spike ${ }^{\mathrm{TM}}$ for Sonic Boom Minimization," AIAA Paper 2008-0124, 2008.

${ }^{6}$ Ordaz, I. and Li, W., "Adaptive Aft Signature Shaping of a Low-Boom Supersonic Aircraft Using Off-Body Pressures," AIAA Paper No. 2012-0020, 2012.

${ }^{7}$ Nadarajah, S., Jameson, A., and Alonso, J., "Sonic Boom Reduction using an Adjoint Method for Wing-Body Configurations in Supersonic Flow," AIAA Paper No. 2002-5547, 2002.

${ }^{8}$ Aftosmis, M., Nemec, M., and Cliff, S., "Adjoint-based Low-Boom Design with Cart3D (Invited)," AIAA Paper No. 2011-3500, 2011.

${ }^{9}$ Jones, W. T., Nielsen, E. J., and Park, M. A., "Validation of 3D Adjoint Based Error Estimation and Mesh Adaptation for Sonic Boom Prediction," AIAA Paper No. 2006-1150, 2006.

${ }^{10}$ Park, M. A., "Low Boom Configuration Analysis with FUN3D Adjoint Simulation Framework," AIAA Paper No. 2011-3337, 2011.

${ }^{11}$ Park, M. A. and Darmofal, D. L., "Validation of an Output-Adaptive, Tetrahedral Cut-Cell Method for Sonic Boom Prediction," AIAA Journal, Vol. 48, No. 9, 2010, pp. 1928-1945.

${ }^{12}$ Park, M. A., Lee-Rausch, E. M., and Rumsey, C. L., "FUN3D and CFL3D Computations for the First High Lift Prediction Workshop," AIAA Paper No. 2011-936, 2011.

${ }^{13}$ Nielsen, E. J., Diskin, B., and Yamaleev, N. K., "Discrete Adjoint-Based Design Optimization of Unsteady Turbulent Flows on Dynamic Unstructured Grids," AIAA Journal, Vol. 48, No. 6, 2010, pp. 1195-1206.

${ }^{14}$ Nielsen, E. and Diskin, B., "Discrete Adjoint-Based Design for Unsteady Turbulent Flows on Dynamic Overset Unstructured Grids," AIAA Paper No. 2012-0554, 2012.

${ }^{15}$ Rallabhandi, S. K., "Sonic Boom Adjoint Methodology and its Applications (Invited)," AIAA Paper No. 2011-3497, 2011.

${ }^{16}$ Rallabhandi, S. K., "Advanced Sonic Boom Prediction Using Augmented Burger's Equation," Journal of Aircraft, Vol. 48, No. 4, 2011, pp. 1245-1253.

${ }^{17}$ Cleveland, R. O., "Propagation of Sonic Booms Through a Real, Stratified Atmosphere," Ph.D. thesis, University of Texas at Austin, 1995.

${ }^{18}$ Conte, S. D. and deBoor, C., Elementary Numerical Analysis, McGraw-Hill, New York, 1972.

${ }^{19}$ Kwon, Y.-H., "Butterworth Digital Filters," http://www.kwon3d.com/theory/filtering/butt.html, accessed June 2012. 
${ }^{20}$ Lyness, J. N., "Numerical Algorithms Based on the Theory of Complex Variables," Proceedings of the ACM 22nd National Conference, 1967, pp. 124-134.

${ }^{21}$ Lyness, J. N. and Moler, C. B., "Numerical Differentiation of Analytic Functions," SIAM Journal on Numerical Analysis, Vol. 4, 1967, pp. 202-210.

${ }^{22} \mathrm{Li}, \mathrm{W}$. and Rallabhandi, S. K., "Inverse Design of Low-Boom Supersonic Concepts Using Reversed Equivalent-Area Targets (Invited)," AIAA Paper No. 2011-3498, 2011.

${ }^{23}$ Nielsen, E. J. et al., "FUN3D: Fully Unstructured Navier-Stokes," http://fun3d.larc.nasa.gov/, accessed June 2012.

${ }^{24}$ Nielsen, E. J. and Jones, W. T., "Integrated Design of an Active Flow Control System Using a Time-Dependent Adjoint Method," Mathematical Modeling of Natural Phenomena, Vol. 6, No. 3, 2011, pp. 141-165.

${ }^{25}$ Kleb, W. L., Nielsen, E. J., Gnoffo, P. A., Park, M. A., and Wood, W. A., "Collaborative Software Development in Support of Fast Adaptive Aerospace Tools (FAAST)," AIAA Paper No. 2003-3978, 2003.

${ }^{26} \mathrm{Li}$, W., Shields, E., and Geiselhart, K., "A Mixed- Fidelity Approach for Design of Low- Boom Supersonic Aircraft," AIAA Paper No. 2010-0845, 2010.

${ }^{27}$ Pirzadeh, S., "Three-Dimensional Unstructured Viscous Grids by the Advancing-Layers Method," AIAA Journal, Vol. 34, No. 1, 1996, pp. 43-49.

${ }^{28}$ Campbell, R. L., Carter, M. B., Deere, K. A., and Waithe, K. A., "Efficient Unstructured Grid Adaptation Methods for Sonic Boom Prediction," AIAA Paper 2008-7327, 2008.

${ }^{29}$ Samareh, J. A., "A Novel Shape Parameterization Approach," Tech. Rep. NASA TM-1999-209116, NASA Langley Research Center, Hampton, VA, May 1999. 2004.

${ }^{30}$ Samareh, J. A., "Aerodynamic Shape Optimization based on free-form deformation," AIAA Paper No. 2004-4630,

${ }^{31}$ Gill, P. E., Murray, W., et al., "NPSOL: A Fortran Package for Nonlinear Programming," http://www. sbsi-sol-optimize.com/, accessed June 2012.

${ }^{32}$ Blue, J., Fox, P., Fullerton, W., et al., "PORT Mathematical Subroutine Library," http://www.bell-labs.com/ project/PORT/, accessed June 2012.

${ }^{33}$ Wrenn, G. A., "An Indirect Method for Numerical Optimization Using the Kreisselmeier-Steinhauser Function," NASA CR 4220, March 1989.

${ }^{34}$ Gilmore, P. and Kelley, C. T., "An Implicit Filtering Algorithm for Optimization of Functions with many Local Minima," SIAM Journal of Optimization, Vol. 5, 1995, pp. 269-285.

${ }^{35}$ Haglund, G. T., "HSCT designs for reduced sonic boom," Proceedings of the AIAA Aircraft Design Systems and Operations Meeting, American Institute for Aeronautics and Astronautics, Sept. 1991. 


\section{Appendix}

The tridiagonal matrices for the relaxation processes are:

$$
\begin{aligned}
& A^{n}, A_{2}^{n}=\left(\begin{array}{cccccc}
1 & 0 & \ldots & & \\
0 & 1 & 0 & \cdots & & \\
0 & -\alpha \kappa_{1}-\kappa_{2} & \left(1+2 \alpha \kappa_{1}\right) & \kappa_{2}-\alpha \kappa_{1} & \cdots & \\
& \ddots & \ddots & \ddots & & \\
& & \ldots & 0 & 1 & 0 \\
& & \ldots & & 0 & 1
\end{array}\right) \\
& B^{n}, B_{2}^{n}=\left(\begin{array}{cccccc}
1 & 0 & \cdots & & \\
0 & 1 & 0 & \cdots & \\
\alpha^{\prime} \kappa_{1}-\kappa_{2} & \left(1-2 \alpha^{\prime} \kappa_{1}\right) & \kappa_{2}+\alpha^{\prime} \kappa_{1} & \cdots & \\
& \ddots & \ddots & \ddots & & \\
& & \cdots & 0 & 1 & 0 \\
& & \cdots & & 0 & 1
\end{array}\right)
\end{aligned}
$$

In the above matrices, $\kappa_{1}=\frac{C_{\nu} \Delta \sigma_{n}}{\Delta \tau^{2}}, \kappa_{2}=\frac{\theta_{\nu}}{2 \Delta \tau}$, and $\alpha^{\prime}=1-\alpha$. If using the Crank-Nicholson scheme, $\alpha=0.5$. For thermo-viscous absorption, the matrices are given below with $\lambda=\frac{\Delta \sigma_{n}}{2 \Gamma(\Delta \tau)^{2}}$

$$
\begin{aligned}
A_{3}^{n}= & \left(\begin{array}{ccccc}
1 & 0 & \ldots & & \\
-\lambda & (1+2 \lambda) & -\lambda & \ldots & \\
& \ddots & \ddots & \ddots & \\
& & \ldots & 0 & 1
\end{array}\right) \\
B_{3}^{n}= & \left(\begin{array}{ccccc}
1 & 0 & \ldots & & \\
\lambda & (1-2 \lambda) & \lambda & \ldots & \\
& \ddots & \ddots & \ddots & \\
& & \ldots & 0 & 1
\end{array}\right)
\end{aligned}
$$

